\title{
Modelling of Quantum Yields in Photocatalytic Membrane Reactors Immobilising Titanium Dioxide
}

\author{
Ignazio Renato Bellobono, ${ }^{1}$ Giulia de Martini, ${ }^{2}$ Paola Maria Tozzi, ${ }^{2}$ Carmen Canevali, ${ }^{3}$ \\ Franca Morazzoni, ${ }^{3}$ Roberto Scotti, ${ }^{3}$ and Riccardo Bianchi ${ }^{4}$ \\ ${ }^{1}$ Environmental Research Centre, University of Milan, Via C. Golgi 19, 20133 Milan, Italy \\ ${ }^{2}$ Research and Development Group, BIT srl, 20121 Milan, Italy \\ ${ }^{3}$ Department of Materials Science, University of Milano Bicocca, 20126 Milan, Italy \\ ${ }^{4}$ CNR, ISTM, 20133 Milan, Italy
}

Received 19 February 2006; Revised 25 April 2006; Accepted 4 May 2006

\begin{abstract}
For some model molecules (methanoic and ethanoic acids, methane, and phenol), systematic investigations of quantum yields were carried out in the present paper, as a function of concentration and of absorbed radiant power. Quantum yields $\Phi_{0}$, calculated from rates, followed an apparently Langmuirian function of initial concentration $C_{0}$, by which $\Phi_{\infty}$ values at "infinite" concentration could be obtained. By having thus established that quantum yields of photomineralisation $\Phi_{\infty}$ are independent of radiation wavelength, within the absorption range of semiconductor, but depend on radiant power, such a dependency was experimentally investigated. For all the investigated molecules, the maximum allowable values reached in the low radiant power range clearly appeared as a plateau. On the contrary, at high radiant power values, another plateau, at a value of about $1 / 4-1 / 5$ with respect to the maximum value, was evident. This was interpreted on the basis of a competition kinetics of hydroxyl radicals with themselves, leading to hydrogen peroxide formation, other than with substrate or intermediate molecules leading to full mineralisation. Modelling of quantum yields as a function of concentration and radiant power thus allows a fully consistent and trustworthy design of photoreactors.
\end{abstract}

Copyright (C) 2006 Ignazio Renato Bellobono et al. This is an open access article distributed under the Creative Commons Attribution License, which permits unrestricted use, distribution, and reproduction in any medium, provided the original work is properly cited.

\section{INTRODUCTION}

Since the 80 s, photocatalysis on semiconductors has been envisaged as one of the most promising methodologies to purify water and air from organic micropollutants [1-8]. The principle, on which photocatalysis is founded, had been discovered and investigated about ten years earlier in energy conversion by photoelectrochemical cells or in semiconductor catalyzed hydrogen photogeneration from water [915]. In both cases, photogenerated holes react with hydroxyl groups of water to give hydroxyl radicals, while photogenerated electrons in deaerated water reduce hydroxonium ions and cause hydrogen evolution. On the contrary, in aerated water, the photogenerated conduction band electrons reduce dissolved oxygen or other oxygen donors, added to this purpose, to yield $\mathrm{O}_{2}{ }^{--}$, in acid-base equilibrium with $\mathrm{HO}_{2}{ }^{\circ}$. The possibility of the latter reaction to take place quantitatively is of uttermost importance in environmental applications of photocatalyis, because, in the last case, oxidation of organic compounds may be carried out by $\mathrm{HO}_{2}{ }^{\bullet}$ together with $\cdot \mathrm{OH}$ radicals. This allows to obtain a fourfold maximum quantum yield with respect to that permissible if each absorbed photon would be able to produce a single hydroxyl radical only. This occurrence has been shown as effective [16-18] when using, for example, ozone as oxygen donor, and when promoting the activity of the semiconductor by suitable doping agents, such as $\mathrm{Co}(\mathrm{III})$ or $\mathrm{V}(\mathrm{V})$, coimmobilised with the semiconductor itself in a membrane structure, and acting as scavengers of generated photoelectrons. On the same side of this problem, it had been suggested theoretically [19] and experimentally proven [20] that the quantum efficiency of oxidation of hydrocarbons dissolved in water on illuminated $n-\mathrm{TiO}_{2}$ was limited by the $\mathrm{O}_{2}$ reduction rate. This semiconductor does not possess a sufficient density of shallow, near-surface electron traps to assist in the $\mathrm{O}_{2}$ reduction process. This causes, in turn, accumulation of electrons on the semiconductor and acceleration of the rate of radiationless electron-hole recombination, a process which heads the loss of the semiconductor photoactivity. On the contrary, incorporation of group VIII metals 
as cocatalysts [20-22] promotes electron transfer to $\mathrm{O}_{2}$ dissolved in water, and thus increases the overall quantum efficiency.

As a consequence, among the major problems in photocatalysis, from the point of view of environmental applications, two aspects readily emerge. First of all, the necessity of evaluating quantum yields, in order to establish if oxidation processes of organic micropollutants are led by ${ }^{\bullet} \mathrm{OH}$ radicals only, and to what extent, or also by other labile oxygen species, such as $\mathrm{HO}_{2}{ }^{\bullet}$ together with ${ }^{\bullet} \mathrm{OH}$ radicals, and to what extent, as it has been underlined above. Secondly, the necessity of reaching the highest performance possible in photocatalytic reactors, in continuous mode, from the engineering point of view [23]. Unfortunately, these two decisive aspects seem to be rather neglected in the literature, and only recently [23] their importance has been emphasized.

In preceding works of this series (see, e.g., among the most recent ones $[18,24,25]$, and references cited therein) the attention has been concentrated primarily on immobilisation of photocatalyst, as well as of its photopromoting agents, acting as scavengers of photogenerated electrons, in a membrane structure, since modular membrane photoreactors are probably the best candidates [23] in terms of performance and energy efficiency. Second, and parallel, great consideration and interest have been directed to the evaluation of quantum efficiencies, particularly by a detailed investigation, at a laboratory-scale level, as a function of concentration and absorbed radiant power, carried out [26] on methanoic and ethanoic acids in aqueous solution. In the present paper, this study has been extended to methane and phenol in aqueous solutions, as model molecules of aliphatic and aromatic compounds, at a laboratory scale for the former and at a pilot plant scale for the latter. Systematic investigations of quantum yields were carried out, as a function of initial concentration of substrates, and of absorbed radiant power, in order to interpret and rationalise the whole matter by adequate modelling, by using both the data of the present work and those of previous paper [26].

\section{EXPERIMENTAL DETAILS}

\subsection{Materials}

Methane and phenol were obtained from Fluka (purity greater than 99.8\%). They were used as received with no further purification. Their aqueous solutions were prepared with ultrapure water (maximum contents of $\mathrm{Na}^{+}$and heavy metal ions 0.02 and $0.004 \mathrm{mg} \mathrm{Kg}^{-1}$, resp.): this was obtained by cross-flow ultrafiltration on composite membranes immobilising active carbon and nuclear grade ion exchange resins, as described [27]. Concentrations of substrates were varied in the range $0.1-10.0 \mathrm{ppm}$ (mass expressed as carbon/volume) for methane and in the range 10.0-1000 ppm for phenol. Stoichiometric hydrogen peroxide was added in all experiments.

\subsection{Photocatalytic membranes}

The photocatalytic membranes (PHOTOPERM BIT/313), kindly supplied by BIT srl, Milan, I, were standard membranes, immobilising $30 \pm 3 \mathrm{wt} \%$ of titanium dioxide (P25 by Degussa, Germany) and no photocatalytic promoter, as cocatalyst. Photocatalytic membranes [28] were described and characterised in previous studies. These membranes were prepared (patents pending) by grafting and graftpolymerising, onto a nonwoven polypropylene tissue, $250 \pm$ $9 \mathrm{gm}^{-2}$ of a prepolymeric blend containing $30 \pm 3 \mathrm{wt} \%$ of titanium dioxide (P25 by Degussa, Germany), in the presence of proprietary photoinitiating and photosensitising systems. Electron micrograph of the surface top layer of one of these membranes is shown in Figure 1. Aggregates of the titanium dioxide nanopowder, anchored and immobilised onto the microporous channels of the membrane, may be clearly observed.

\subsection{Apparatus, procedures, and modelling}

Experiments with methane solutions, in a laboratory-scale photoreactor, were carried out exactly as illustrated in [25]. The only difference in the procedure was that all sets of experiments, each of them as a function of initial concentration of substrate $C_{0}$, were repeated by varying the radiating power in each set. A high-pressure mercury arc lamp with a nominal power of $0.50 \mathrm{~kW}$ was employed, kindly supplied by BIT srl (Milan, Italy): it was further provided with a step-by-step power regulation device, so that power absorbed by the membrane, as determined actinometrically, could be varied between 0.10 and $4.35 \mathrm{Wcm}^{-1}$. The membrane being concentric to the lamp in the photoreactor used, absorbed power per unit length of lamp was also the same, if referred to unit length of membrane. At $0.30 \mathrm{Wcm}^{-1}$ the overall power absorbed by the membrane, within the absorption range of immobilised semiconductor, in the experimental conditions of the present work, was $30 \mathrm{~W}$, corresponding to $4.45 \times 10^{-5}$ Einstein $\mathrm{s}^{-1}$. This same ratio between absorbed power per unit length of membrane and photon flux was of course operative at lower and higher values of absorbed power. In this paper, as well as in all preceding papers of this series, when the concerned laboratory-scale experiments were carried out in the presence of hydrogen peroxide as oxygen donor, the radiating flux, unless otherwise stated, being filtered by a suitable borosilicate glass, corresponded to a radiation wavelength range from the band gap of $\mathrm{TiO}_{2}$ downwards to $315 \mathrm{~nm}$, so that direct photolysis of hydrogen peroxide to give hydroxyl radicals was inhibited. Owing to the refrigeration system of the lamp, by means of water circulating in the photoreactor, mean temperature during the runs was $308 \pm 2 \mathrm{~K}$.

Experiments with phenol solutions, in a pilot plant-scale photoreactor, equipped with 9 membrane modules, were carried out exactly as described in [18]. The only difference in the procedure was that all sets of experiments, each of them as a function of initial concentration of substrate, were repeated by varying the radiating power in each set, and by 


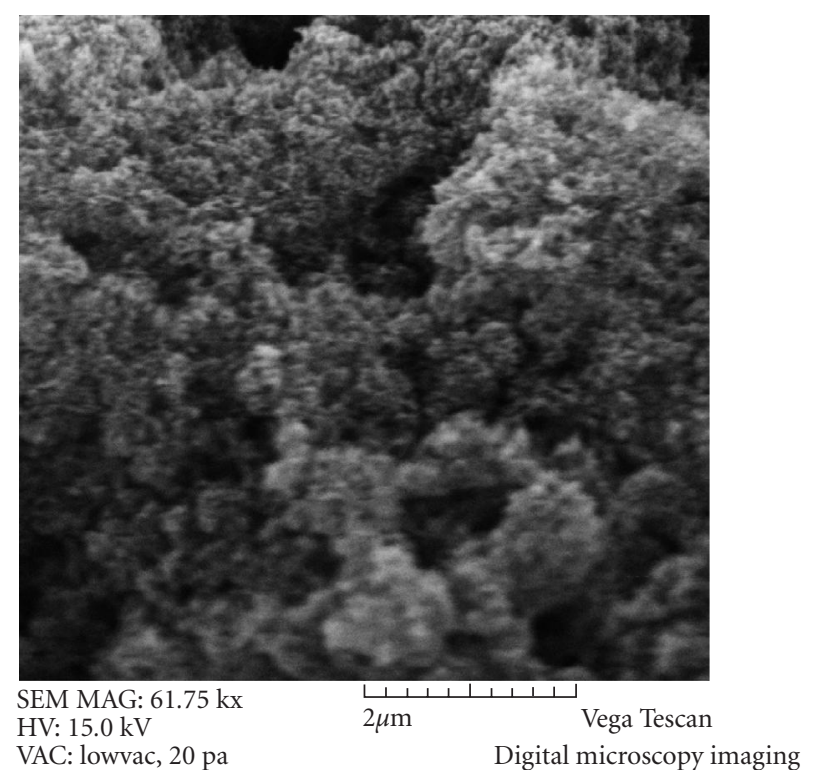

Date: 10/11/05

Name: TiO-4

Figure 1: Electron micrograph (scale shown on figure) of the surface top layer of a membrane $\left(\right.$ PHOTOPERM ${ }^{\circledR}$ BIT/313) prepared by photoinitiated grafting and graft-polymerisation onto a nonwoven polypropylene tissue of $250 \mathrm{gm}^{-2}$ of a prepolymeric blend containing $30 \pm 3 \mathrm{wt} \%$ of titanium dioxide (P25 by Degussa, Germany) in the presence of proprietary photoinitiating and photosensitising systems.

using in each module the same kind of lamps described in the preceding paragraph, relative to methane experiments. In all kinds of experiments, both at a laboratory-scale and at a pilot plant-scale level, the diameter of the photocatalytic membrane was $5.8 \pm 0.1 \mathrm{~cm}$.

In the present, as well as in previous papers of this series, membranes were placed and fixed coaxially with the lamp, either in laboratory-scale or pilot plant-scale photoreactors in such a way that the flow was perpendicular to the membrane. Owing to the microporous structure of the membrane (see Section 2.2), the pressure drop was negligible: it corresponded to $0.8-1.5 \mathrm{~mm} \mathrm{Hg}$ when the flow rate was $2 \mathrm{~m}^{3} / \mathrm{h}$ in each module.

In each set of experiments, the absorbed radiant power per unit length of photocatalytic membrane was first fixed at some chosen value in the range $0.10-4.4 \mathrm{Wcm}^{-1}$, and the disappearance of substrate, as well as the total organic carbon (TOC) mineralisation, were followed kinetically, as a function of $C_{0}$. To interpret experimental data the kinetic model was employed $[24,25]$ by which mineralisation of substrate to $\mathrm{CO}_{2}$ was supposed to occur by kinetic constants $k_{1}$ through one single intermediate mediating the behaviour of all the numerous real intermediates formed in the path from the substrate to $\mathrm{CO}_{2}$ (kinetic constants of formation of the latter being $k_{2}$ ). A competitive Langmuirian adsorption of both substrate and "intermediate" was also supposed to be operative, as expressed by apparent adsorption constants $K_{1}$ and $K_{2}$, possessing a, partly at least, kinetic significance. By Langmuir-Hinshelwood treatment of initial rate data, starting values of the $k$ and $K$ couples were obtained, from which, by a set of differential equations $[24,25]$, the final optimised parameters, $k_{1}$ and $K_{1}, k_{2}$ and $K_{2}$, were calculated, able to fit the whole photomineralisation curve, and not only its initial segment, as the Langmuirian parameters do. In particular, $K_{1}$ and $K_{2}$ showed very similar values, and very often coincided, within the limits of experimental and modelling uncertainty. By using the optimised parameters above, the rates of photomineralisation were evaluated as a function of initial concentration, and consequently, by knowing the photon flux, the quantum yields $\Phi_{0}$ were obtained. Quantum yields $\Phi_{0}$, calculated from rates, followed an apparently Langmuirian function of initial concentration $C_{0}$, by which $\Phi_{\infty}$ values at "infinite" concentration could be obtained:

$$
\frac{1}{\Phi_{0}}=\frac{1}{\Phi_{\infty}}+\left(\frac{1}{\Phi_{\infty} C_{0} K}\right),
$$

where the $K$ value substantially coincided with the $K_{2}$ constant of the four-parameter kinetic model above for all the molecules investigated.

Finally, by repeating all sets of experiments, at different values of the absorbed radiant power per unit length of photocatalytic membrane, which was varied in the range 0.10 $4.4 \mathrm{Wcm}^{-1}$, and by applying (1) to each set of these experiments, the $\Phi_{\infty}$ values as a function of the absorbed radiant power, per unit length of photocatalytic membrane, were obtained.

\section{RESULTS AND DISCUSSION}

Photomineralisation of methane in aqueous solution, in a laboratory-scale reactor, and in a concentration range corresponding to $0.10-10.0 \mathrm{ppm}$ (mass/volume) of carbon, as well as of phenol in aqueous solution, in a pilot plant-scale reactor, and in a concentration range corresponding to 10.0$1000 \mathrm{ppm}$ (mass/volume) of carbon, was studied at $308 \pm$ $2 \mathrm{~K}$ by polychromatic irradiation with a photon flux, which was varied in the range $0.10-4.4 \mathrm{~W} / \mathrm{cm}$ (see Section 2), in the presence of stoichiometric hydrogen peroxide as oxygen donor, by photocatalytic membranes immobilising $30 \pm 3 \%$ of titanium dioxide. Kinetics of both substrate disappearance, to yield intermediates, and total organic carbon (TOC) disappearance, to yield carbon dioxide, were followed.

By employing a kinetic model based on four parameters, thoroughly described in preceding papers (see, e.g., [24]), mineralisation of substrate to $\mathrm{CO}_{2}$ was supposed to occur through one single intermediate mediating the behaviour of all possible intermediates formed in the way from the substrate to $\mathrm{CO}_{2}$. This also implies that both substrate and simulating "intermediate" showed a competitive apparent Langmuirian adsorption onto the immobilized semiconductor, as expressed by apparent adsorption constants $K_{1}$ and $K_{2}$, respectively, while the kinetic constants $k_{1}$ and $k_{2}$ articulated the degradation of substrate and "intermediate," respectively. When both $k$ and $K$ couples, for substrate and "intermediate," are accessible experimentally, as in the present work, 


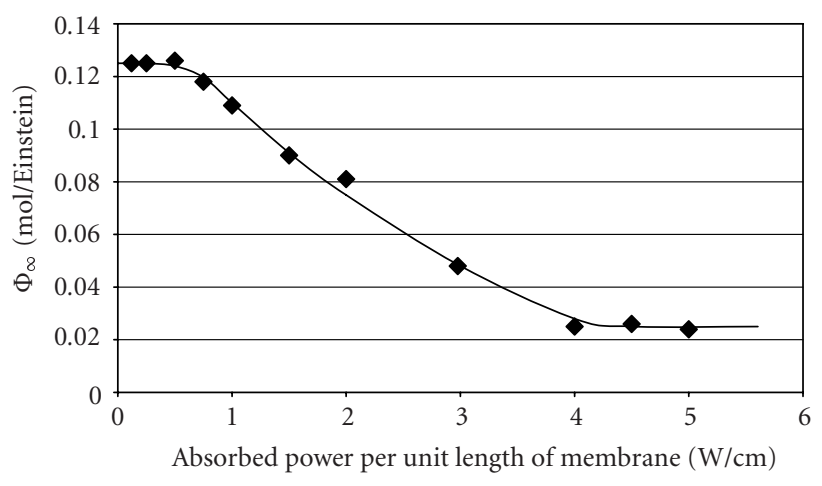

Figure 2: Quantum efficiencies $\Phi_{\infty}$ (mol/Einstein), extrapolated by (1) (see Section 2), at "infinite" concentration of substrate for methane in aqueous solution as a function of absorbed power per unit length of irradiated photocatalytic membrane expressed as $\mathrm{Wcm}^{-1}$.

that is, when initial rate data, relative to substrate, and TOC as well, have been measured, they may be first treated by the classical Langmuir-Hinshelwood equation. The respective values drawn by the Langmuirian model are unable to describe the whole curve of mineralisation. They may be used, however, as starting values of the model above to draw from the differential equations of this model an optimisation of the whole temporal kinetic curves of substrate and TOC until consistency is reached and the best fitting of experimental curves is obtained.

By using the optimised parameters above, the rates of photomineralisation were evaluated as a function of initial concentration $C_{0}$ at a fixed value of photon flux, and consequently, by knowing the photon flux from the ratio of the rate to the photon flux, the quantum yields $\Phi_{0}$ corresponding to $C_{0}$ were determined. Quantum yields $\Phi_{0}$, calculated from rates, followed an apparently Langmuirian function of initial concentration $C_{0}$, as expressed by (1) (see Section 2). Through this equation, $\Phi_{\infty}$ values at "infinite" concentration could be obtained. By these sets of experiments, and by finally varying the photon flux in each set, the experimental dependency of $\Phi_{\infty}$ on the absorbed radiation power per unit length of photocatalytic membrane, expressed in $\mathrm{Wcm}^{-1}$ (see Section 2) could be evaluated.

The $K$ values obtained for methane and phenol, by linear regression of (1) (see Section 2), were $0.098 \pm 0.003$ (ppm $\mathrm{C})^{-1}$ and $0.035 \pm 0.001(\mathrm{ppm} \mathrm{C})^{-1}$, respectively, where the uncertainties are expressed as probable errors. The $K$ value practically coincides, for methane, with the value of $K_{2}$, or with an intermediate value between $K_{1}$ and $K_{2}$ expressed in the same units as obtained from modelling of experimental data by the four-parameter kinetic model in [25]. For phenol too, the $K$ value practically coincides with $K_{1} \sim K_{2}$, expressed in the same units, as obtained from the analogous modelling of experimental data [18].

From the previous data concerning the methanoic and ethanoic acids [26], the $K$ values following (1), $0.010 \pm 0.001$ $(\mathrm{ppm} \mathrm{C})^{-1}$ and $0.026 \pm 0.001(\mathrm{ppm} \mathrm{C})^{-1}$, respectively,

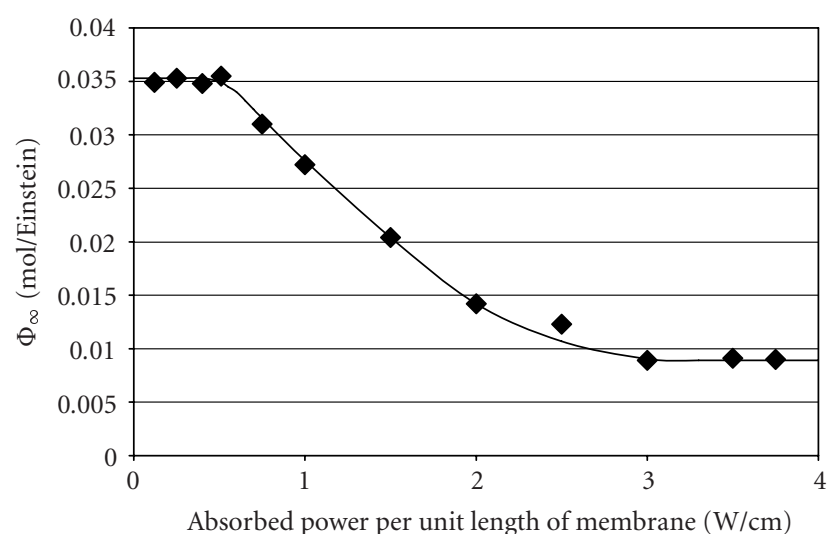

FIGURE 3: Quantum efficiencies $\Phi_{\infty}$ (mol/Einstein), extrapolated by (1) (see Section 2), at "infinite" concentration of substrate for phenol in aqueous solution as a function of absorbed power per unit length of irradiated photocatalytic membrane expressed as $\mathrm{Wcm}^{-1}$.

similarly coincide with the mean values of $K_{1} \sim K_{2}$, or their mean, the latter representing apparent thermodynamic constants [26] for the four-parameter kinetic modelling which has been carried out for these molecules [26].

From this coincidence, as well as from the analytical form of (1), it may immediately be inferred that quantum yields $\Phi_{0}$ represent the photochemical counterparts of kinetic constants, in the Langmuirian sense, and thus follow the same competition function between interfacial electronic transfer and recombination of charge carriers onto the semiconductor surface.

Furthermore, by (1) (see Section 2), the extrapolated values of $\Phi_{\infty}$ as a function of absorbed radiation power per unit length of membrane can be evaluated, as reported in Figures 2, 3, 4, and 5, for methane, phenol, methanoic acid, and ethanoic acid, respectively.

In Figures 2-5, some common features may be immediately remarked. Firstly, two plateaux of nearly constant $\Phi_{\infty}$ values appear in these curves: the former at low values of photon flux, and the latter at high values of this flux. The higher values of these limiting $\Phi_{\infty}$ plateaux substantially coincide, within the limits of experimental and modelling uncertainty, with the maximum allowable quantum yields $\Phi_{\max }$ evaluated as the reciprocal of the number of hydroxyl radicals imposed by stoichiometry to mineralise one molecule of substrate, in the hypothesis that the mineralisation process is carried out by ${ }^{\bullet} \mathrm{OH}$ radicals only. These $\Phi_{\max }$ values amount, respectively, to $0.125,0.0357,0.50,0.11 \mathrm{~mol} /$ Einstein for methane, phenol, methanoic acid, and ethanoic acid, respectively. The values of the second $\Phi_{\infty}$ plateau, corresponding to the high photon fluxes area, on the contrary, are lower than the maximum allowable quantum yields $\Phi_{\max }$, by a factor of around 1/4-1/5 (1/3.95 for phenol). At intermediate photon fluxes, transitional values between the two plateaux are observed.

In order to enlighten and explain this behaviour, a competition kinetics of the reaction of hydroxyl radicals with themselves to give hydrogen peroxide, accompanied by the 


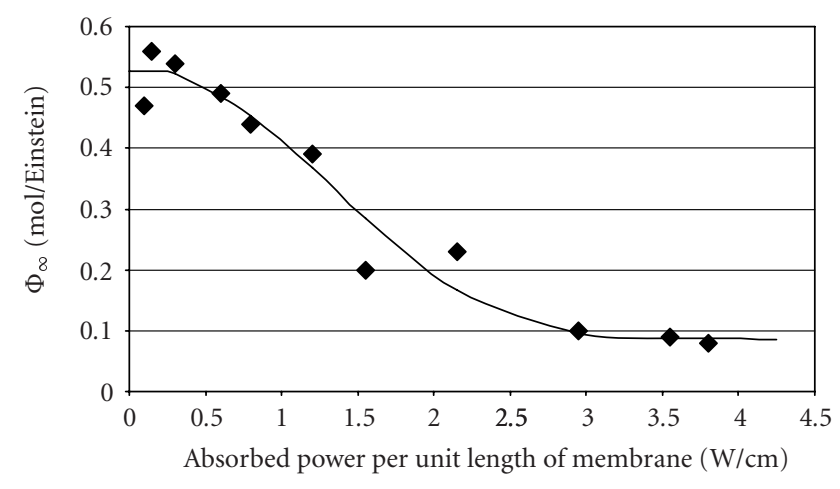

Figure 4: Quantum efficiencies $\Phi_{\infty}$ (mol/Einstein), extrapolated by (1) (see Section 2), at "infinite" concentration of substrate for methanoic acid in aqueous solution as a function of absorbed power per unit length of irradiated photocatalytic membrane expressed as $\mathrm{Wcm}^{-1}$. Data taken from [26].

reaction of these radicals leading to mineralisation of substrate may be envisaged, that given by reactions (a) and (b):

$$
\cdot \mathrm{OH}+{ }^{\bullet} \mathrm{OH} \longrightarrow \mathrm{H}_{2} \mathrm{O}_{2}
$$

the rate of which $r_{a}$ may be written as

$$
r_{a}=k_{a} \cdot C_{\cdot \mathrm{OH}}^{2}
$$

where $k_{a}$ is the rate constant of reaction $(\mathrm{a})$, and $C \cdot \mathrm{OH}$ the concentration of hydroxyl radicals:

$$
\cdot \mathrm{OH}+\text { substrate (or intermediates) } \longrightarrow \text { mineralisation }
$$

the rate of which $r_{b}$ may be written as

$$
r_{b}=k_{b} \cdot C_{S} \cdot C \cdot \mathrm{OH}
$$

where $k_{b}$ is the rate constant of reaction (b), and $C_{S}$ is the concentration of substrate or intermediates on the surface of the photocatalytic membrane onto which mineralisation takes place.

At low photon fluxes, at which the concentration of hydroxyl radicals formed by irradiation onto the semiconductor surface is relatively low, reaction (a) is certainly negligible with respect to reaction (b), so that a quantum efficiency equal or very near to the maximum one allowable may be attained. Conversely, when photon fluxes increase above a certain value, the competition kinetics may be the operative mechanism at an overall rate $r$ given by

$$
r=r_{a}+r_{b}
$$

and a mineralisation yield (effective quantum efficiency) given by a factor $r_{b} / r$ of the maximum allowable quantum efficiency. In order for this factor to possibly work, two conditions are needed. Scavenging of photogenerated electrons of the conduction band $\mathrm{e}^{-} \mathrm{CB}$ should be quantitative, and it should not give rise to species, radicals, or others, interfering

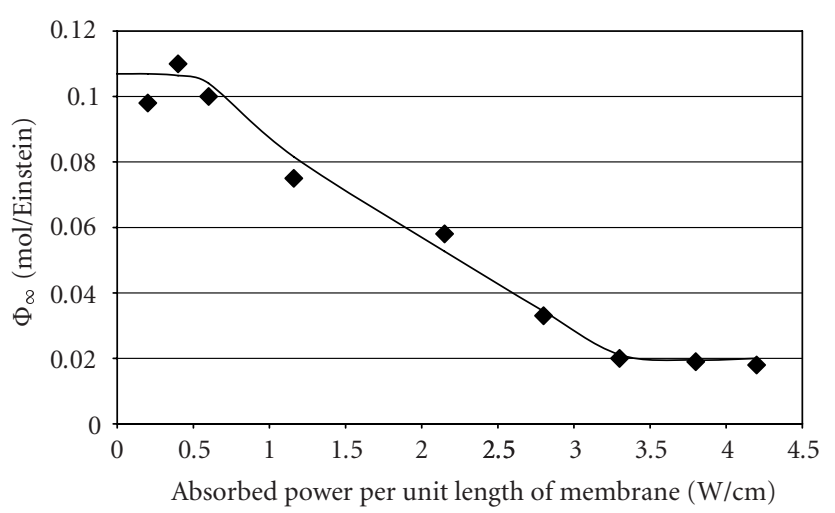

FIgURE 5: Quantum efficiencies $\Phi_{\infty}$ (mol/Einstein), extrapolated by (1) (see Section 2), at "infinite" concentration of substrate for ethanoic acid in aqueous solution as a function of absorbed power per unit length of irradiated photocatalytic membrane expressed as $\mathrm{Wcm}^{-1}$. Data taken from [26].

with reactions (a) and (b), or able to vary quantum efficiency with respect to the maximum value, the latter being given by the production of hydroxyl radicals solely by reaction of water with holes as stated above. This kind of scavenging is compatible with reactions (c) and (d) occurring at comparable rates, and followed by reaction (e):

$$
\begin{gathered}
\mathrm{e}^{-} \mathrm{CB}+\mathrm{H}_{2} \mathrm{O}_{2} \longrightarrow{ }^{\bullet} \mathrm{OH}+\mathrm{OH}^{-}, \\
\mathrm{e}^{-} \mathrm{CB}^{+}+\mathrm{O}_{2} \longrightarrow \mathrm{O}_{2}^{\bullet-} / \mathrm{HO}_{2}{ }^{\bullet}, \\
\mathrm{HO}_{2}{ }^{\bullet} \mathrm{OH} \longrightarrow \mathrm{O}_{2}+\mathrm{H}_{2} \mathrm{O} .
\end{gathered}
$$

If these reactions take place quantitatively, in the order given, radical reactivity is controlled by (4), the $r_{b} / r$ factor reaching the maximum value permissible (approaching the unity) when $r_{a}$ is much less than $r_{b}$, a situation achieved at very low values of the photon flux.

In order to verify the suitableness of kinetic model defined by (2)-(4) above, also in the condition of high photon fluxes, when the $r_{b} / r$ factor of the maximum allowable quantum efficiency corresponds to the lower plateau in curves such as those of Figures 2-5, the $k_{b}$ values should be determined for the photocatalytic membrane reaction corresponding to (3), given that the $k_{a}$ value $\left(4.3 \times 10^{9} \mathrm{M}^{-1} \cdot \mathrm{s}^{-1}\right)$ may be reasonably accepted to be independent on the support, and is known from the literature [29]. Relative to the four molecules examined in the present paper, the $k_{b}$ value $\left(1.4 \times 10^{10} \mathrm{M}^{-1} \cdot \mathrm{s}^{-1}\right)$ has been measured for $\mathrm{TiO}_{2}$ immobilised in photocatalytic membranes, only with phenol as substrate [30]. Consequently, for this case, the ability of (2)(4) to fit experimental data of Figure 3 may be checked, by considering that $\Phi_{0}$, and therefore $\Phi_{\infty}$ values are determined in limiting conditions of $C_{0} \rightarrow \infty$, and an acceptable physical condition for $C_{0} \rightarrow \infty$ corresponds to $C_{0}$ values of $10^{-3}$ $10^{-2} \mathrm{M}$. By assuming, in this range of values, that concentration of hydroxyl radicals is about tenfold with respect to the substrate concentration, that is, $10^{-2}-10^{-1} \mathrm{M}$, respectively, which is quite reasonable in the experimental conditions of the high photon fluxes used, by (2)-(4), a plateau value of 


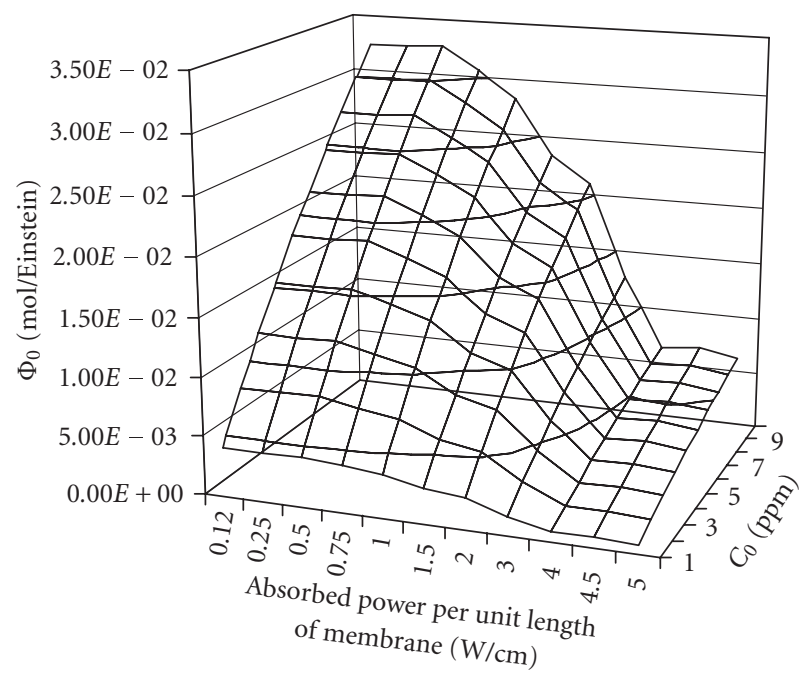

Figure 6: Quantum yields $\Phi_{0}$ (mol of methane/Einstein) as a function of initial concentration $C_{0}$ expressed as ppm (mass/volume) of carbon and of absorbed power per unit length of lamp (and membrane) expressed as $\mathrm{Wcm}^{-1}$.

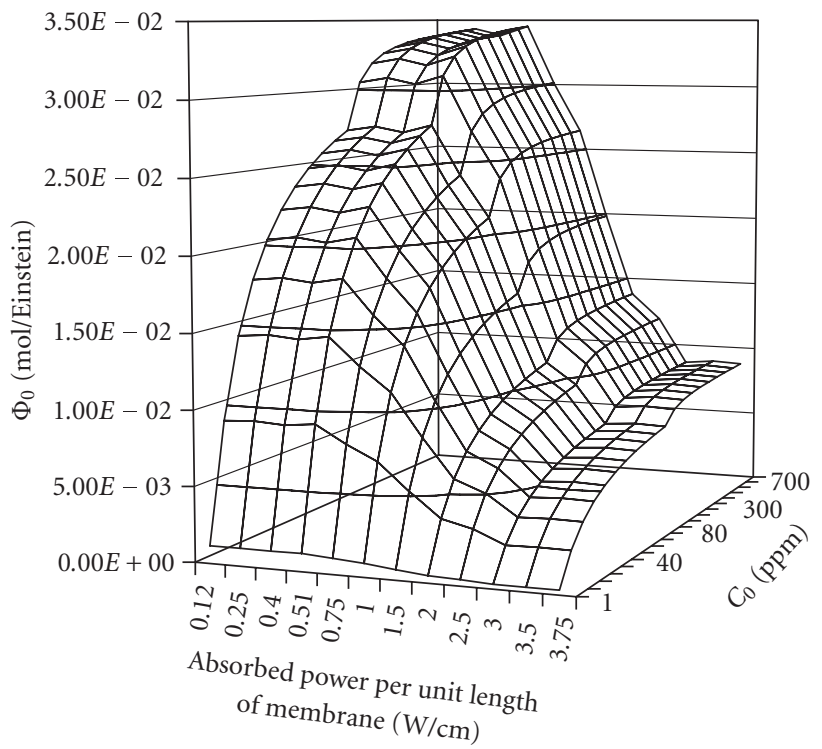

Figure 7: Quantum yields $\Phi_{0}$ ( $\mathrm{mol}$ of phenol/Einstein) as a function of initial concentration $C_{0}$ expressed as ppm (mass/volume) of carbon and of absorbed power per unit length of lamp (and membrane) expressed as $\mathrm{Wcm}^{-1}$.

about $1 / 4$, with respect to $\Phi_{\max }$, may be calculated, in perfect agreement with value of Figure 3. To conclude, the kinetic model above is fully consistent with experimental data both in the low and in the high range of photon fluxes. Furthermore, the small variability of the $r_{b} / r$ factors, shown in Figures $2-5$, ranging from $1 / 4$ to $1 / 5$, even for a small group of molecules, but with a big difference in chemical nature, from aliphatic to aromatic compounds, may originate from

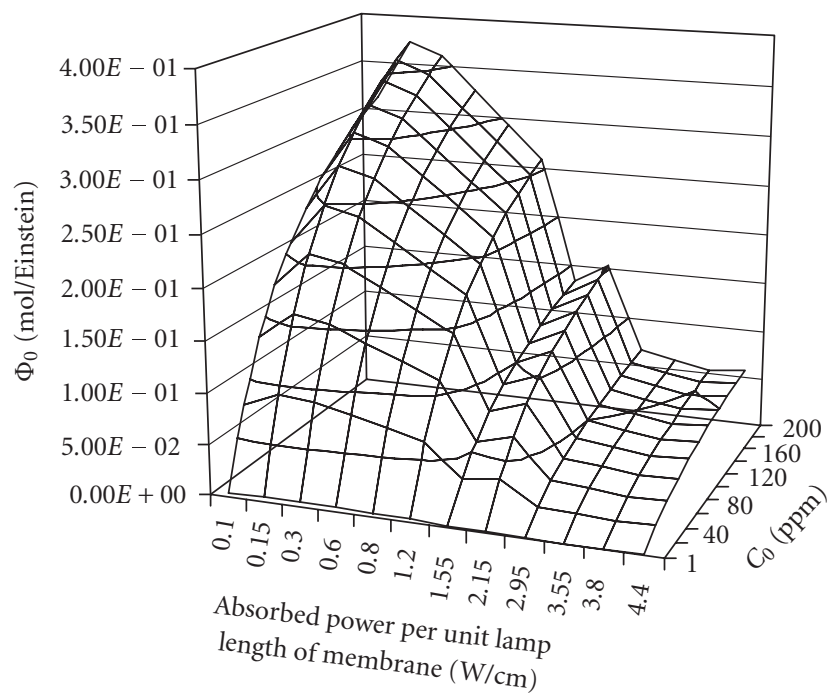

FIgURE 8: Quantum yields $\Phi_{0}$ (mol of methanoic acid/Einstein) as a function of initial concentration $C_{0}$ expressed as ppm (mass/vol ume) of carbon and of absorbed power per unit length of lamp (and membrane) expressed as $\mathrm{Wcm}^{-1}$.

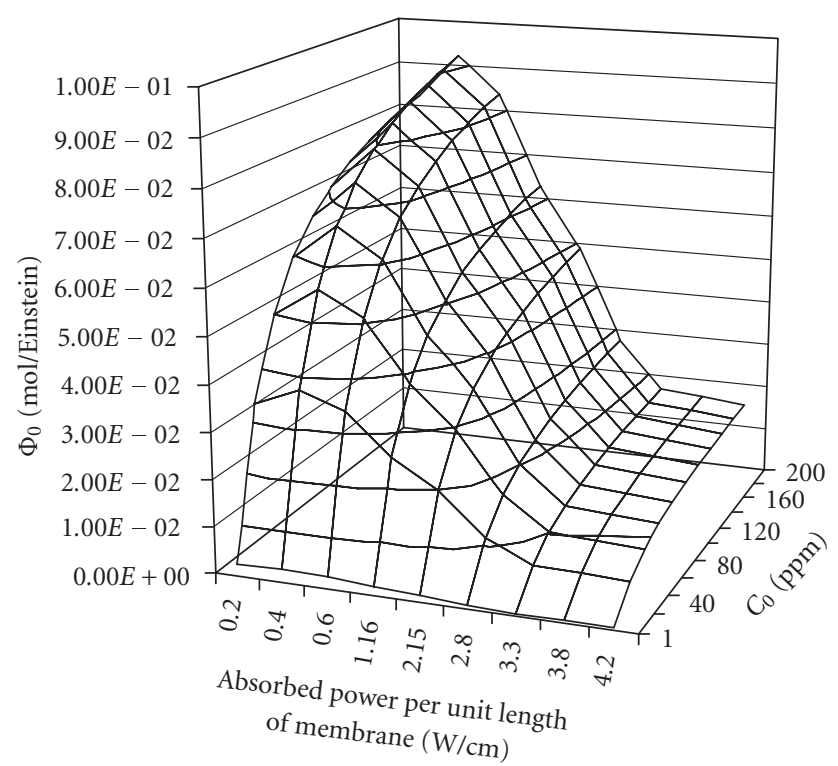

Figure 9: Quantum yields $\Phi_{0}$ (mol of ethanoic acid/Einstein) as a function of initial concentration $C_{0}$ expressed as ppm (mass/vol ume) of carbon and of absorbed power per unit length of lamp (and membrane) expressed as $\mathrm{Wcm}^{-1}$.

the fact that the $k_{b}$ values [30] are very near to $10^{10} \mathrm{M}^{-1} \cdot \mathrm{s}^{-1}$, and change very moderately within an approximate factor of 0.5 to 2 , with change of the chemical structure.

Finally, by combining (1) with the graphs of the curves of Figures $2-5$, complete modelling of quantum yields of photomineralisation as a function of $C_{0}$ and of absorbed radiant power per unit length of membrane is possible, as shown in Figures 6, 7, 8, and 9 for methane, phenol, methanoic acid, 
and ethanoic acid in aqueous solutions, respectively. These curves, of course, display, in the planes of constant concentration, the behaviour of curves of Figures 2-5 discussed above. In the planes of constant absorbed power per unit length of membrane, on the contrary, Langmuirian-type performance, reaching "saturation" at high concentration values, evidences the four-parameter kinetic model used to interpret and rationalise rates over the whole mineralisation reaction profiles. To sum up, modelling of quantum yields as a function of concentration of substrate undergoing mineralisation, and of radiant power per unit length of photocatalytic membrane thus allows a fully consistent and trustworthy design of the membrane photoreactors.

\section{CONCLUSIONS}

Quantum yields of mineralisation of substrates in annular photoreactors immobilising the photocatalyst in a membrane structure show a Langmuirian-type dependency on initial concentration of substrates themselves, by which limiting quantum yields, corresponding to "infinite" concentration may be calculated, together with an apparent thermodynamic constant coinciding with the corresponding parameters of a kinetic modelling based on four parameters, and able to fit the whole kinetic curve up to complete mineralisation.

These limiting quantum yields depend on radiant power absorbed per unit of membrane length in the cylindrical geometry of photoreactors. In the low radiant power range they take the shape of a plateau corresponding to the maximum allowable quantum yields, thus showing the excellent performance of the photocatalytic membrane reactors. On the contrary, at high radiant power values, another plateau is evident, at a value of about $1 / 4-1 / 5$ with respect to the maximum value. This was interpreted on the basis of a competition kinetics of hydroxyl radicals with themselves leading to hydrogen peroxide formation, other than with substrate or intermediates molecules leading to full mineralisation.

By combining the dependency of quantum yield from concentration with that of limiting quantum yields on radiant power absorbed per unit length of membrane, comprehensive modelling of quantum yields as a function of concentration of substrate and radiant power may be satisfactorily obtained.

\section{ACKNOWLEDGMENTS}

The current paper is the 77th in a series of papers authored by Ignazio Renato Bellobono and his collaborators over the past 20 years which are collectively referred to as the "Photosynthetic Membranes" series. Financial contribution by Fondazione Cariplo is gratefully acknowledged.

\section{REFERENCES}

[1] A. L. Pruden and D. F. Ollis, "Photoassisted heterogeneous catalysis: the degradation of trichloroethylene in water," Journal of Catalysis, vol. 82, no. 2, pp. 404-417, 1983.

[2] H. Y. Hsiao, C. L. Lee, and D. F. Ollis, "Heterogeneous photocatalysis: degradation of dilute solutions of dichloromethane
$\left(\mathrm{CH}_{2} \mathrm{Cl}_{2}\right)$, chloroform $\left(\mathrm{CHCl}_{3}\right)$, and carbon tetrachloride $\left(\mathrm{CCl}_{4}\right)$ with illuminated $\mathrm{TiO}_{2}$ photocatalyst," Journal of Catalysis, vol. 82, no. 2, pp. 418-423, 1983.

[3] T. Nguyen and D. F. Ollis, "Complete heterogeneously photocatalyzed transformation of 1,1- and 1,2-dibromoethane to carbon dioxide and hydrogen bromide," Journal of Physical Chemistry, vol. 88, no. 16, pp. 3386-3388, 1984.

[4] R. W. Matthew, "Hydroxylation reactions induced by nearultraviolet photolysis of aqueous titanium dioxide suspensions," Journal of the Chemical Society, Faraday Transactions 1, vol. 80, pp. 457-471, 1984.

[5] R. W. Matthew, "Photo-oxidation of organic material in aqueous suspensions of titanium dioxide," Water Research, vol. 20, no. 5, pp. 569-578, 1986.

[6] K. Okamoto, Y. Yamamoto, H. Tanaka, M. Tanaka, and A. Itaya, "Heterogeneous photocatalytic decomposition of phenol over $\mathrm{TiO}_{2}$ powder," Bulletin of the Chemical Society of Japan, vol. 58, pp. 2015-2022, 1985.

[7] M. Barbeni, E. Pramauro, E. Pelizzetti, E. Borgarello, M. Grätzel, and N. Serpone, "Photodegradation of 4-chlorophenol catalyzed by titanium dioxide particles," Nouveau Journal De Chimie, vol. 8, pp. 547-550, 1984.

[8] H. Al-Ekabi and N. Serpone, "Kinetic studies in heterogeneous photocatalysis. 1. Photocatalytic degradation of chlorinated phenols in aerated aqueous solutions over $\mathrm{TiO}_{2}$ supported on a glass matrix," Journal of Physical Chemistry, vol. 92, no. 20, pp. 5726-5731, 1988.

[9] A. Fujishima and K. Honda, "Electrochemical photolysis of water at a semiconductor electrode," Nature, vol. 238, pp. 3738, 1972.

[10] A. Fujishima, K. Kohayakawa, and K. Honda, "Hydrogen production under sunlight with electrochemical photo-cell," Journal of the Electrochemical Society, vol. 122, p. 1487, 1975.

[11] A. J. Nozik, "Photoelectrolysis of water using semiconducting $\mathrm{TiO}_{2}$ crystals," Nature, vol. 257, pp. 383-386, 1975.

[12] M. S. Wrighton, D. S. Ginley, P. T. Wolczanski, A. B. Ellis, D. L. Morse, and A. Linz, "Photoassisted electrolysis of water by irradiation of a titanium dioxide electrode," Proceedings of the National Academy of Sciences of the United States of America, vol. 72, no. 4, pp. 1518-1522, 1975.

[13] J. Manassen, D. Cahen, G. Hodes, and A. Sofer, "Electrochemical, solid state, photochemical and technological aspects of photoelectrochemical energy converters," Nature, vol. 263, pp. 97-100, 1976.

[14] W. Gissler, P. L. Lensi, and S. Pizzini, "Electrochemical investigation of an illuminated $\mathrm{TiO}_{2}$-electrode," Journal of Applied Electrochemistry, vol. 6, no. 1, pp. 9-13, 1976.

[15] B. Marcandalli and I. R. Bellobono, "Influence of wavelength on the photoeffects at polycrystalline semiconductors. Energy conversion efficiencies of photoelectrochemical cells employing a titanium dioxide film anode and a hydrogen or oxygen cathode," Atti della Accademia Nazionale dei Lincei, Rendiconti, Classe di Scienze Fisiche, Matematiche e Naturali, vol. 65, pp. 87-92, 1978.

[16] F. Gianturco, C. M. Chiodaroli, I. R. Bellobono, M. L. Raimondi, A. Moroni, and B. Gawlik, "Pilot-plant photomineralization of atrazine in aqueous solution, by photocatalytic membranes immobilising titanium dioxide and promoting photocatalysts," Fresenius Environmental Bulletin, vol. 6, no. 78, pp. 461-468, 1997.

[17] L. Rivas, I. R. Bellobono, and F. Ascari, "Photomineralization of $n$-alkanoic acids in aqueous solution by photocatalytic membranes. Influence of trialkyl vanadates as catalytic 
promoters of immobilized titanium dioxide," Chemosphere, vol. 35, no. 12, pp. 2889-2908, 1997.

[18] I. R. Bellobono, F. Ascari, C. Lagrasta, et al., "Kinetic modelling of photomineralization of phenol, as model molecule of aromatic micropollutants, and validation of a photochemical reactor based on photocatalytic membranes immobilizing titanium dioxide and promoting photocatalysts," Fresenius Environmental Bulletin, vol. 12, no. 12, pp. 1536-1544, 2003.

[19] H. Gerischer and A. Heller, "Photocatalytic oxidation of organic molecules at $\mathrm{TiO}_{2}$ particles by sunlight in aerated water," Journal of the Electrochemical Society, vol. 139, no. 1, pp. 113-118, 1992.

[20] C. M. Wang, A. Heller, and H. Gerischer, "Palladium catalysis of $\mathrm{O}_{2}$ reduction by electrons accumulated on $\mathrm{TiO}_{2}$ particles during photoassisted oxidation of organic compounds," Journal of the American Chemical Society, vol. 114, no. 13, pp. 5230-5234, 1992.

[21] J. Chen, D. F. Ollis, W. H. Rulkens, and H. Bruning, "Photocatalyzed oxidation of alcohols and organochlorides in the presence of native $\mathrm{TiO}_{2}$ and metallized $\mathrm{TiO}_{2}$ suspensions. Part (I): photocatalytic activity and $\mathrm{pH}$ influence," Water Research, vol. 33, no. 3, pp. 661-668, 1999, 1173.

[22] A. Dobosz and A. Sobczyński, "Water detoxification: photocatalytic decomposition of phenol on $\mathrm{Au} / \mathrm{TiO}_{2}$," Monatshefte für Chemie, vol. 132, no. 9, pp. 1037-1045, 2001.

[23] M. F. J. Dijkstra, E. C. B. Koerts, A. A. C. M. Beenackers, and J. A. Wesselingh, "Performance of immobilized photocatalytic reactors in continuous mode," American Institute of Chemical Engineers Journal, vol. 49, no. 3, pp. 734-744, 2003.

[24] F. Ascari, I. R. Bellobono, and P. M. Tozzi, "Kinetic modelling of pilot plant photomineralization of aqueous cibacron CR as model molecule of industrial azo dyes on titanium dioxideimmobilizing membranes," Fresenius Environmental Bulletin, vol. 12, no. 10, pp. 1195-1201, 2003.

[25] I. R. Bellobono, F. Morazzoni, R. Bianchi, et al., "Laboratoryscale photomineralisation of $n$-alkanes in aqueous solution, by photocatalytic membranes immobilising titanium dioxide," International Journal of Photoenergy, vol. 7, no. 2, pp. 79-85, 2005.

[26] L. Rivas, I. R. Bellobono, and F. Ascari, "Photomineralization of $n$-alkanoic acids in aqueous solution by photocatalytic membranes. Influence of radiation power," Chemosphere, vol. 37, no. 6, pp. 1033-1044, 1998.

[27] I. R. Bellobono, R. Tacchi, E. Selli, and F. Muffato, "Photosynthetic membranes for pure and ultrapure water production," in Membrane Separation Processes, A. Green, Ed., p. 187, BHRA, Cranfield, UK, 1989.

[28] B. Barni, A. Cavicchioli, E. Riva, et al., "Laboratory-scale photodegradation of phenol in aqueous solution by photocatalytic membranes immobilizing titanium dioxide," Chemosphere, vol. 30, no. 10, pp. 1847-1860, 1995.

[29] A. J. Elliot and D. C. Ouellete, "Temperature dependence of the rate constant for the reaction e-aq+ $\mathrm{OH}$ in water up to $150{ }^{\circ} \mathrm{C}$," Journal of the Chemical Society, Faraday Transactions, vol. 90, pp. 837-841, 1994.

[30] R. Morelli, I. R. Bellobono, C. M. Chiodaroli, and S. Alborghetti, "EPR spin-trapping of hydroxyl radicals onto photocatalytic membranes immobilizing titanium dioxide, and spin adduct competition, as a probe of reactivity with aqueous organic micropollutants," Journal of Photochemistry and Photobiology A: Chemistry, vol. 112, no. 2, pp. 271-276, 1998. 


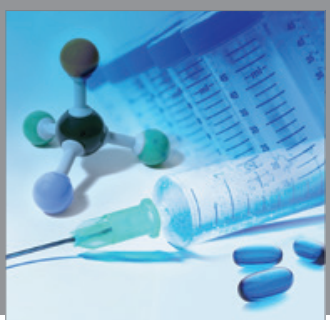

International Journal of

Medicinal Chemistry

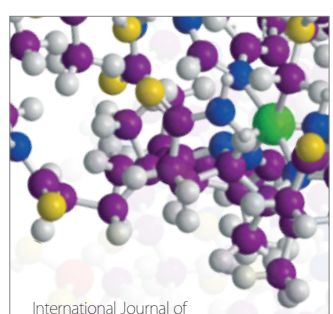

Carbohydrate Chemistry

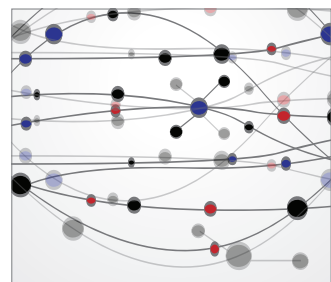

The Scientific World Journal
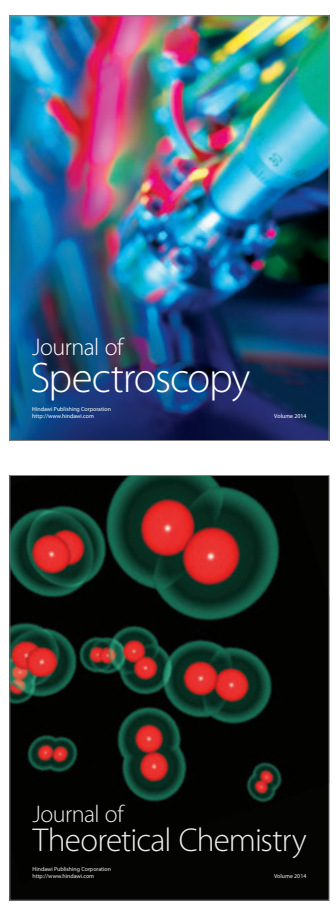
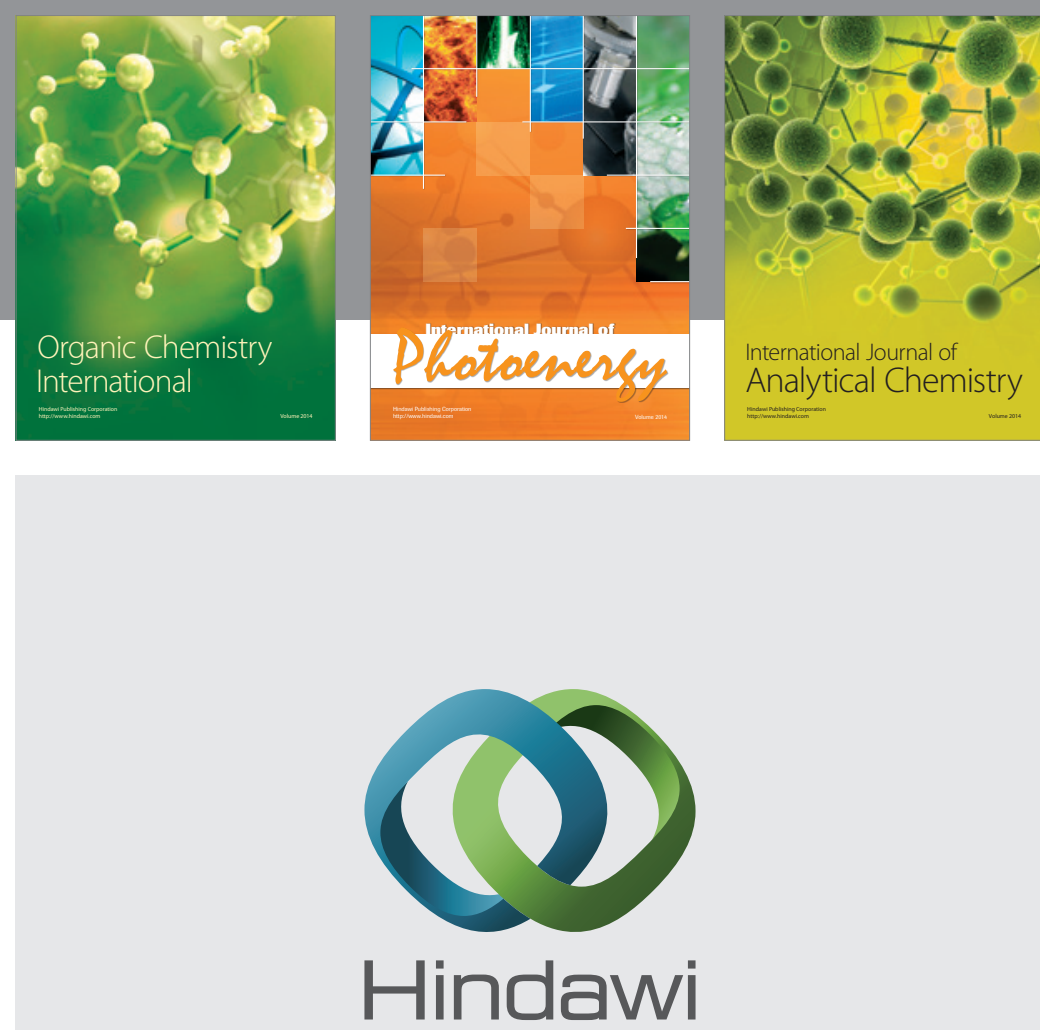

Submit your manuscripts at

http://www.hindawi.com
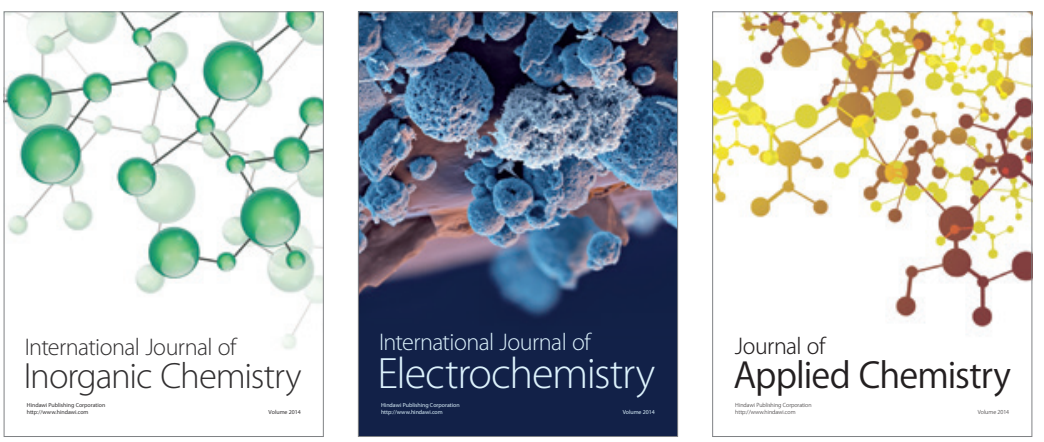

Journal of

Applied Chemistry
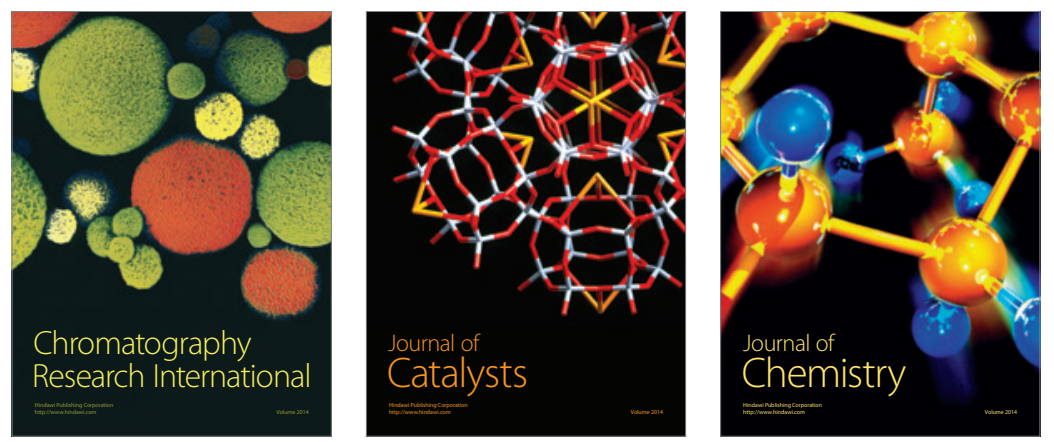
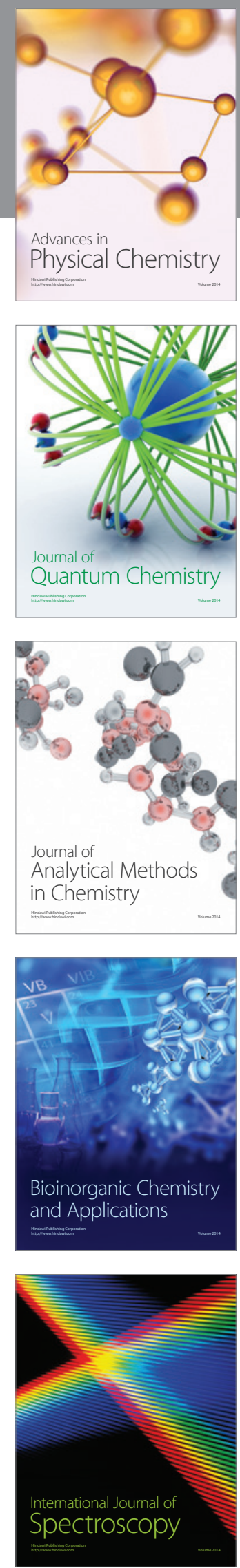